\title{
2016-2017 Reviewers-Consultants 2016-2017
}

The following individuals have graciously provided reviews for the Canadian Journal of Experimental Psychology during the period of November 2016 to November 2017. We thank them for their thoughtful and timely assistance.

De novembre 2016 à novembre 2017, les personnes suivantes ont offert leur collaboration spéciale dans l'évaluation de manuscrits pour la Revue canadienne de psychologie expérimentale. Nous les remercions de leur aide précieuse.

\begin{tabular}{|c|c|c|c|}
\hline Naseem Al-Aidroos & Peter Garrard & Anne Lafay & Daniel Richardson \\
\hline Nicole Anderson & Lupita Estefania Gazzo & Jason P. Leboe-McGowan & Katherine M. Robinson \\
\hline \multirow{2}{*}{ Mark Ashcraft } & Castañeda & Karen Li & \\
\hline & Amanda Gilchrist & Baptist Liefooghe & Bennett L. Schwartz \\
\hline Clément Belletier & Peter Graf & Jason Lodge & Wolfgang Schwarz \\
\hline Elina Birmingham & & & Alexander Shackman \\
\hline Caroline Blais & Aroline Hanson & Pedro Macizo & Judith M. Shedden \\
\hline Julie Bouchard & Thierry Hasbroucq & Yaniv Mama & David Sidhu \\
\hline Thomas Bullock & Heather Henderson & Heath Matheson & Scott Sinnett \\
\hline Deb Burke & Richard Henson & Diana Mazzarella & Marcia Spetch \\
\hline Thomas Busey & Thomas Hills & Beat Meier & Jennifer A. Stolz \\
\hline \multirow{4}{*}{$\begin{array}{l}\text { Caroline Cellard } \\
\text { Craig Chapman } \\
\text { Jim Clark } \\
\text { Alexandre Coutté }\end{array}$} & William E. Hockley & Douglas Mewhort & David Strayer \\
\hline & Kathleen L. Hourihan & Corinne Molinaro & Bradley Sturz \\
\hline & Mark J. Huff & $\begin{array}{l}\text { Catherine Monnier } \\
\text { Laura Morett }\end{array}$ & Karl Szpunar \\
\hline & Mike Humphreys & Weimin Mou & Tracy L. Taylor-Helmick \\
\hline \multirow{3}{*}{$\begin{array}{l}\text { Amedeo D’Angiulli } \\
\text { Sidney D'Mello } \\
\text { Jason Droll }\end{array}$} & Michal Icht & Ian Neath & Barbara Tillman \\
\hline & Roxane Itier & Antie Nuthmann & Sébastien Tremblay \\
\hline & Jason Ivanoff & & Chi-Shing Tse \\
\hline \multirow[t]{2}{*}{ Lisa Emery } & Debra Jared & Daniel Oppenheimer & Paul Verhaeghen \\
\hline & Tanya R. Jonker & Jean-Charles Pelland & Troy Visser \\
\hline Myra Fernandes & & Gordon Pennycook & John R. Vokey \\
\hline Daniel Fiset & Solene Kalenine & Curtis E. Phills & \\
\hline \multirow[t]{2}{*}{ John Franchak } & Mike Kalish & Jerome Prado & Scott Watter \\
\hline & Pentti Kanerva & Steven Prime & Tim Welsh \\
\hline Mathieu Gagnon & Jun I. Kawahara & Mary A. Pyc & \\
\hline Giovanni Galfano & Raymond Klein & & \\
\hline Xiaoqing Gao & Peter Kwantes & Kimberly Quinn & \\
\hline
\end{tabular}

